\title{
Inicio de la época reproductiva y tiempo de defensa del territorio en machos de Colinus leucopogon (Galliformes: Odontophoridae)
}

\author{
Luis Sandoval \\ Escuela de Biología, Universidad de Costa Rica, San Pedro de Montes de Oca. 11501-2060, San José, Costa Rica; \\ biosandoval@hotmail.com
}

\author{
Recibido 05-VII-2010. Corregido 30-IX-2010. Aceptado 30-X-2010.
}

\begin{abstract}
Reproductive season onset and time invested in territory defense in Colinus leucopogon males (Galliformes: Odontophoridae). The factors that determine the onset of the reproductive season and the relationship between territory defense and mating success of Colinus leucopogon males are unknown. Here I report on climatic variables influencing the time of permanence on the territory, and how this affects the species mating success. I also analyze the relationship between the time devoted by males on territory defense and the relationship of song and territorial characteristics. The onset of the reproductive season was determined by an amount of rain greater than $14.3 \mathrm{~mm}$ during March, favouring the food availability and nesting places abundance, and also allowed an increase in the reproductive success of Colinus leucopogon. The time invested in territory defense by males was not related with their mating success. Moreover, the duration in territory defense was similar for males that paired, compared with those that did not. In addition, song and territory characteristics were not related with males invested time in their territory defense. Therefore, this could be another reason explaining the lack of a relationship between the duration in the territories by males and pair formation, and suggests that song characteristics strongly influence the formation of pairs in this species. Rev. Biol. Trop. 59 (1): 363-372. Epub 2011 March 01.
\end{abstract}

Key words: Spot-bellied Bobwhite, rain season, mating success, territory behaviour, reproduction, song.

La fenología de las aves tropicales está influenciada principalmente por los periodos secos y lluviosos (Stiles 1983, 1992, Bancroft et al. 2000, Bosque et al. 2004), y la duración del fotoperiodo (Wikelski et al. 2000). Estos dos factores ambientales influyen (precipitación) y predicen (duración del día) la disponibilidad de alimento (Stiles 1983, Loiselle \& Blake 1990, Poulin et al. 1992, Bancroft et al. 2000, Wikelski et al. 2000), el cual es el recurso limitante en la reproducción de las especies, como por ejemplo en el colibrí Phaetornis longirostris (Stiles 1992), las palomas Patagioenas leucocephala (Bancroft et al. 2000), Columbina talpacoti y C. minuta (Bosque et al. 2004), y el hormiguero Hylophilax naevioides (Wikelski et al. 2000). La temperatura es otro de los factores ambientales que ha sido analizado en estudios fenológicos de aves, pero tiene poca influencia debido a que posee un ámbito de variación pequeño a lo largo del año en los bosques tropicales (Voous 1950, Miller 1965, Poulin et al. 1992, Barrantes \& Sánchez 2003).

En las codornices neotropicales del género Colinus, los factores ambientales que influyen con el inicio de la época reproductiva son poco conocidos, pero parecen estar asociados con el inicio de la estación lluviosa tanto en Costa Rica (Leber 1975), como en Venezuela (Pérez 2000). Durante la época no reproductiva de $C$. leucopogon, las bandadas están conformadas por 4 a 20 individuos de ambos sexos (obs. pers., Leber 1975, Madge \& McGowan 2002), pero al inicio de la época reproductiva, las bandadas se desmembran, y los machos inician la defensa de su territorio (Leber 1975, Sandoval 
2008). Los machos de ésta codorniz emiten vocalizaciones para atraer hembras (canto) las cuales son específicas del inicio y a lo largo del periodo reproductivo, lo que permite con un alto grado de confianza determinar el inicio y el final de la época reproductiva, para una población. Además, dado que los machos cantan únicamente cuando no están apareados, es posible determinar el tiempo de permanencia de cada macho dentro de su territorio.

El tiempo (en semanas) que permanecen los machos de C. leucopogon dentro de sus territorios podría ser una característica importante en la formación de parejas, debido a que el tiempo invertido en la defensa territorial y en la realización de despliegues, son actividades que consumen gran parte de la energía de los machos durante el periodo reproductivo (Vehrencamp et al. 1989, Andersson 1994). Los machos de esta codorniz cantan en promedio 2.2 $\pm 1.4( \pm \mathrm{DS})$ cantos/min y aproximadamente $8 \mathrm{hr}$ al día (datos sin pub.), por lo que permanecer en su territorio cantando por mayor cantidad de semanas, puede ser un indicativo de la reserva energética del macho, característica importante en la atracción de hembras (Vehrencamp et al. 1989). Con el fin de comprender los factores que explican la reproducción en C. leucopogon, los objetivos de este trabajo son (1) determinar si la temperatura y la precipitación están relacionados con el inicio de la época reproductiva de los machos de C. leucopogon, (2) determinar si el tiempo de permanencia de los machos dentro del territorio influye en la adquisición de parejas, y (3) analizar si hay una relación entre las características del canto del macho y la calidad del territorio con la permanencia de los machos dentro de éstos.

\section{MATERIALES Y MÉTODOS}

Área de estudio: La investigación se realizó en Getsemaní, provincia de Heredia, Costa Rica $\left(10^{\circ} 01^{\prime} \mathrm{N}, 84^{\circ} 06^{\prime} \mathrm{W}\right)$, entre los 1240 a 1360 m.s.n.m, en un área de 30ha. La precipitación anual es de $2374 \mathrm{~mm}$, con un periodo seco de diciembre a marzo (Fig. 1), y una temperatura promedio de $20.1^{\circ} \mathrm{C}$. La vegetación del área de estudio estuvo compuesta en un $40 \%$ por plantaciones de café con sombra y un $60 \%$ de potreros. Ambos ambientes estaban divididos por cercas vivas. Aunque fuera de la época reproductiva es posible encontrar esta especie en los cafetales, los machos únicamente defienden territorios en las zonas de potreros.

Muestreo: Se realizaron dos censos semanales de las 07:00 a las 11:00hr a lo largo de un transecto lineal de $3 \mathrm{~km}$ que recorre toda el área de estudio, durante los meses de marzo de 2003 al 2007 para determinar la fecha en que se inició la época reproductiva de C. leucopogon. Observaciones preliminares en el área de estudio indicaban que la época reproductiva de esta codorniz inicia en el mes de marzo. El punto de inicio del recorrido se alternó semanalmente para evitar algún efecto de la hora del muestreo sobre la detectabilidad de los machos de C. leucopogon, ya que los machos disminuyen su actividad de canto conforme pasan las horas después del amanecer, lo que los hace menos detectables. Como indicador del inició de la época reproductiva se utilizó el día cuando los machos comenzaron a cantar (las hembras no cantan), ya que es la única época del año en la que cantan (Leber 1975, Sandoval 2008). Durante cada censo se registró la temperatura promedio, mínima y máxima del día de muestreo, y la precipitación acumulada desde el 1 de marzo hasta el día del muestreo. Los valores de estas variables ambientales se obtuvieron de la estación meteorológica que el Instituto Meteorológico Nacional de Costa Rica tiene en Santa Lucía-Heredia, contiguo al área de estudio $\left(10^{\circ} 01^{\prime} \mathrm{N}, 84^{\circ} 06^{\prime} \mathrm{W}\right)$. Además, de marzo a octubre de 2005 y 2006 , se realizó un censo semanal a lo largo del mismo transecto, para contabilizar el número total de machos territoriales presentes en el área de estudio, la fecha en que cada uno se estableció en su territorio, el tiempo que permaneció en el territorio, el éxito en la adquisición de pareja y la presencia de nidos o pichones.

Se definió como macho territorial a aquel individuo que cantó constantemente durante 


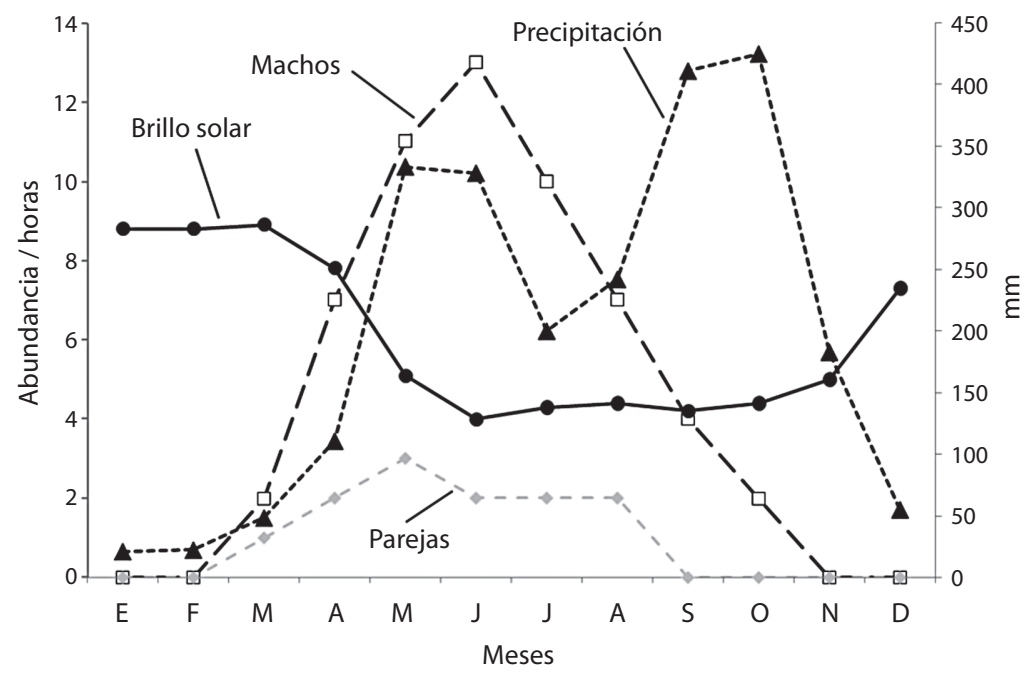

Fig. 1. Número total de machos territoriales presentes cada mes y de parejas de Colinus leucopogon observadas por mes durante los periodos reproductivos de 2005-2006 (marzo a octubre). Precipitación (1982-2006), y horas de brillo solar mensual (1982-2000) en el área de estudio, basado en datos de la estación meteorológica de Santa Lucia de Barva, Heredia, Instituto Meteorológico Nacional de Costa Rica.

Fig. 1. Total territorial males of Colinus leucopogon each month and monthly pair, during 2005 and 2006 reproductive season (March to October). Monthly precipitation (1982-2006), and sun shine hours (1982-2000) in the study area data from Santa Lucia Meteorology Station, Heredia, Costa Rica, Instituto Meteorológico Nacional de Costa Rica.

al menos 5min, y que se desplazó menos de $5 \mathrm{~m}$ alrededor de la percha principal de canto. Aunque no se anillaron los machos para su identificación individual, se asumió como el mismo individuo aquel que se encontró en la misma percha de canto o en una muy cercana, como ha sido propuesto por Seddon \& Tobias (2007), Barrantes et al. (2008) y Fitzsimmons et al. (2008). Si en alguna visita no se escuchó u observó al macho en su territorio, se reprodujo la vocalización de otro macho durante $5 \mathrm{~min}$ : si no respondió, se reprodujo la llamada de una hembra durante $5 \mathrm{~min}$. Observaciones preliminares han demostrado que las codornices territoriales tienden a responder a las grabaciones de los con-específicos si están presentes en su territorio y la grabación se reproduce dentro o cerca del territorio (Eitniaer et al. 2001, Bonaccorso \& Barreto 2002, Hale 2006, Sandoval 2008). Si un macho de C. leucopogon no se observó durante tres semanas seguidas, se consideró que abandonó su territorio. Si el macho no respondió durante una o dos semanas pero sí en la siguiente, la o las semanas que estuvo ausente fue incluida en el periodo total de duración del macho dentro de su territorio.

Se realizó una búsqueda exhaustiva de 30 a 40min cada semana dentro del territorio del macho para determinar si había adquirido pareja. Se consideró que un macho se emparejó si se le observó con una hembra dentro de su territorio y el macho no cantaba.

Medidas del canto: Para determinar si el canto de los machos de C. leucopogon influyó en su permanencia en el territorio, se grabó el canto de cada macho territorial durante la primera semana que se le escuchó cantando. Los cantos se grabaron durante $5 \mathrm{~min}$ utilizando una grabadora Sony TCM-5000EV (versión para aves) y un micrófono direccional Sennheiser ME 66, a distancias entre los 5 y $15 \mathrm{~m}$ del 
individuo focal. Del total de cantos grabados durante los $5 \mathrm{~min}$, se analizaron un máximo de cinco cantos seleccionados de forma aleatoria. Si se grabaron cinco o menos cantos se analizaron todos los cantos disponibles. Los cantos se digitalizaron a una tasa de muestreo de $44100 \mathrm{~Hz}$ y una resolución de 16 bits con el programa Cool Edit 20001.0 (Johnston 1999) y se utilizó el programa Raven 1.2 (Charif et al. 2004) para medir en cada canto (Fig. 2): la frecuencia más baja, la frecuencia más alta, la máxima frecuencia, la diferencia entre la frecuencia baja y la más alta (ámbito de frecuencia), y la duración. Todas estas medidas se realizaron en la frecuencia fundamental del canto, debido a que es en ésta donde se acumula la máxima energía (Catchpole \& Slater 1995). Se calculó un promedio de cada variable del canto por macho para realizar los análisis estadísticos posteriores. Las grabaciones se depositaron en el Laboratorio de Bioacústica de la Escuela de Biología de la Universidad de Costa Rica.

Medidas del territorio: Para comparar las características del territorio entre machos durante la época reproductiva, se utilizó como territorio un área de $25 \mathrm{~m}$ de radio alrededor de la percha principal de canto. Ésta es la mitad de la distancia mínima entre dos machos

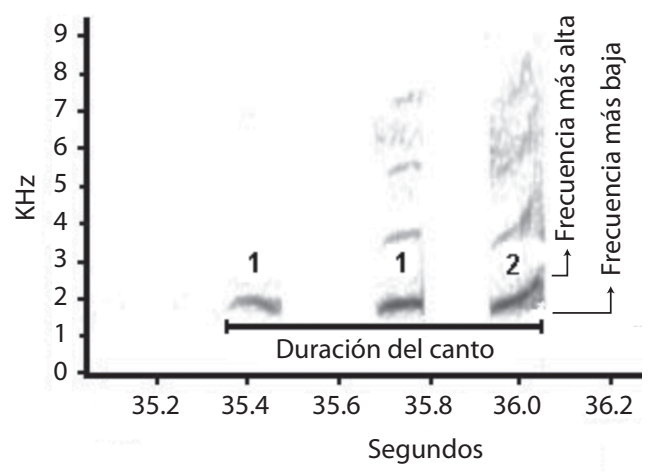

Fig. 2. Canto del macho de Colinus leucopogon, donde se muestran los elementos ( 1 y 2 ) que conforman el canto y las medidas tomadas.

Fig. 2. Colinus leucopogon male song, the song elements (1 and 2) and measures in song are showed. territoriales contiguos (Leber 1975, Sandoval 2008). Dentro de cada territorio se anotó el porcentaje de área potencialmente utilizable para anidar, definida como agrupaciones de pasto de $25 \mathrm{~cm}$ de alto y $30 \mathrm{~cm}$ de diámetro, que son características mínimas para que se construyan nidos (Leber 1975, Stiles \& Skutch 1989). Asimismo, se contabilizó el número de posibles perchas de canto, como rocas expuestas, árboles caídos y arbustos, mayores a $10 \mathrm{~cm}$ y menores a $5 \mathrm{~m}$.

Para analizar el efecto de los valores de temperatura y precipitación sobre el inicio de la época reproductiva se realizó una regresión logística por pasos para comparar los valores de los días donde no hubo machos cantando, con el día en que comenzaron a cantar. Otra regresión logística se utilizó para comparar si la permanencia dentro de los territorios de los machos que adquirieron pareja fue diferente de la de aquellos machos que no se emparejaron.

Para determinar si el tiempo de permanencia de los machos en su territorio, estuvo influenciado por el mes en que se establecieron, se realizó un análisis de varianza de una vía. Con una correlación de rangos de Spearman se compararon las características del canto y el territorio de cada macho, contra la duración en su territorio. Para establecer si la formación de parejas y el número de machos territoriales presentes cada mes varió se realizaron dos pruebas de Kolmogorov-Smirnov de una muestra. Los valores presentados son promedios $\pm \mathrm{DS}$.

\section{RESULTADOS}

Durante el periodo de estudio de 2005 y 2006 se localizaron 30 machos territoriales de C. leucopogon, 15 en cada año. En el 2005 los machos establecieron territorios de marzo hasta setiembre, pero dos machos se mantuvieron presentes hasta octubre. En el 2006 los machos se establecieron y estuvieron presentes de abril hasta agosto. La abundancia de machos territoriales para cada mes durante ambos años no varió significativamente $\left(\mathrm{D}_{\max }=0.37\right.$, $\mathrm{N}=8, \mathrm{p}=0.52$ ); sin embargo, hubo una mayor abundancia de machos en la mitad de la época 
reproductiva (mayo a agosto) (Fig. 1). La formación de parejas ocurrió únicamente de marzo hasta agosto, siendo más abundante en mayo $(n=3)$, mientras que en setiembre y octubre no se encontraron parejas $\left(\mathrm{D}_{\max }=0.63\right.$, $\mathrm{N}=8, \mathrm{p}=0.002$, Fig. 1). De las seis parejas con pichones que se observaron en total, tres ocurrieron en junio y tres en julio.

El periodo reproductivo de C. leucopogon de 2003 al 2007 inició en marzo (Fig. 1), y de los valores de temperatura (máxima, mínima y promedio) y precipitación analizados, solo este último se determinó como la variable ambiental que influyó en el inicio de la época reproductiva (regresión logística: $\chi^{2}=20.70$, $\left.\mathrm{gl}=1, \mathrm{p}<0.001, \mathrm{rho}^{2}=0.74\right)$. La precipitación promedio acumulada necesaria para que iniciara la época reproductiva fue de $34.1 \mathrm{~mm}$, con una mínima de $14.3 \mathrm{~mm}$ y una máxima de 68.9mm (Fig. 3).

La duración promedio de los machos en su territorio fue de $3 \pm 5$ semanas, con una
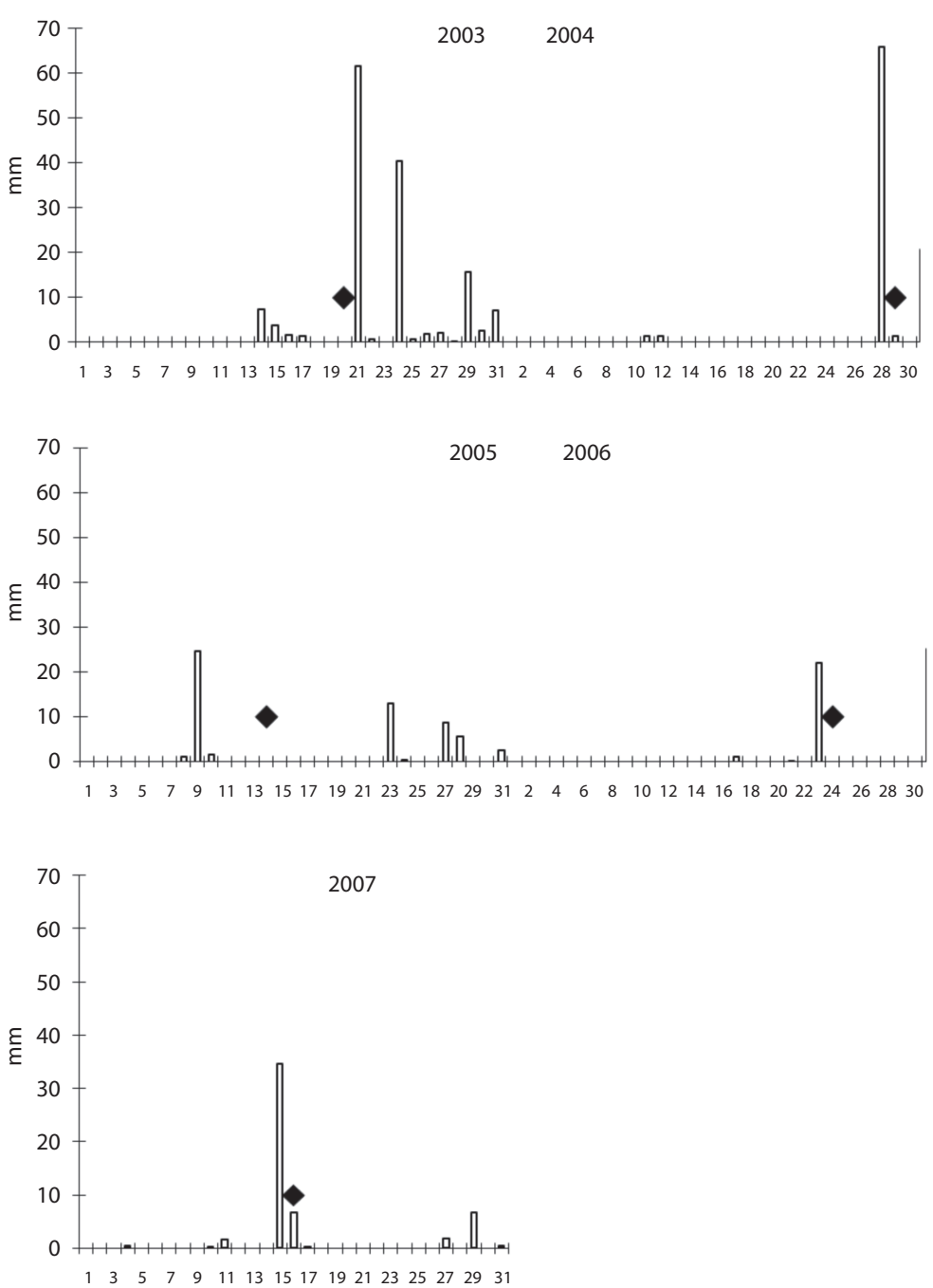

Fig. 3. Precipitación diaria durante el mes de Marzo en cinco años, y la fecha cuando se observó el primer macho de Colinus leucopogon cantando (punto).

Fig. 3. March daily precipitation in five years, and the day when observed the first Colinus leucopogon male sing (point). 


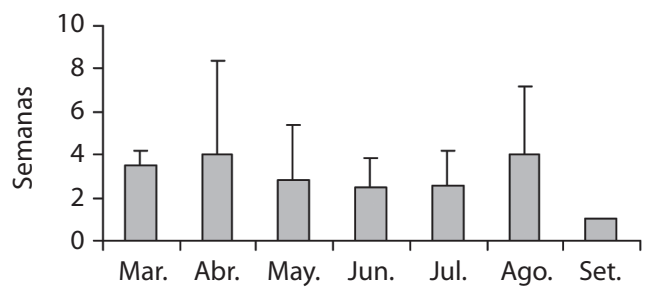

Fig. 4. Duración promedio (+DS) de los machos de Colinus leucopogon dentro de sus territorios, según el mes en que se establecieron dentro de su territorio, durante los periodos reproductivos de 2005-2006.

Fig. 4. Average (+DS) in duration of Colinus leucopogon in its territories based in the month when the male begun to sing during 2005 and 2006 reproductive season.

duración mínima de 1 semana y una máxima de 9 semanas. El mes en que un macho se estableció en su territorio no influyó en el tiempo que permaneció cantando en él $(\mathrm{F}=0.39$, $\mathrm{gl}=6,24, \mathrm{p}=0.88$, Fig. 4). Tampoco influyeron las características territoriales como abundancia de rocas $\left(r_{s}=0.01\right)$, arbustos $\left(r_{s}=-0.14\right)$, y troncos caídos $\left(\mathrm{r}_{\mathrm{s}}=-0.17\right)$, o el área potencial para anidación $\left(\mathrm{r}_{\mathrm{s}}-0.16\right)$; ni los valores de frecuencia del canto (baja: $r_{s}=0.07$, alta: $r_{s}=-0.13$, máxima: $\mathrm{r}_{\mathrm{s}}=0.16$, ámbito: $\left.\mathrm{r}_{\mathrm{s}}=-0.10\right)$, y duración $\left(r_{\mathrm{s}}=0.25\right) \quad(\mathrm{p}>0.05$ para todas las comparaciones; Fig. 5). Los machos que adquirieron parejas, permanecieron la misma cantidad de semanas en su territorio que los que no se emparejaron $(2.82 \pm 1.47$ y $2.95 \pm 2.61$ semanas respectivamente) (regresión logística: $\chi^{2} 0.24$, $\mathrm{gl}=1, \mathrm{p}=0.62, \mathrm{rho}^{2}=0.006$ ).

\section{DISCUSIÓN}

El inicio del ciclo reproductivo de C. leucopogon concordó con el fin del periodo seco y el inicio del lluvioso, y se asoció directamente con un aumento en la precipitación durante marzo, y no con valores de la temperatura diaria. Esto concuerda con estudios previos en aves que habitan los bosques tropicales, donde se ha encontrado que los ciclos fenológicos están marcados por la estacionalidad de los periodos secos y lluviosos (Stiles 1983, 1992; Loiselle \& Blake 1990, Bancroft et al. 2000, Bosque et al. 2004). El aumento en la precipitación se ha asociado a un incremento en el alimento (Leber 1975, Stiles 1983, Loiselle \& Blake 1990, Bancroft et al. 2000, Lusk et al. 2001), lo que puede favorecer la reproducción de C. leucopogon, porque existe una mayor disponibilidad de semillas e insectos para los adultos y las crías. Una variable ambiental que no se midió, pero que podría estar influyendo en el inicio del periodo reproductivo de C. leucopogon, es la duración diaria del fotoperiodo (Wikelski et al. 2000). Sin embargo esta variable ambiental no se incluyó debido a que no existen los datos para el periodo del estudio (Fig. 1). Además datos mensuales de horas de brillo solar tomados en la zona de estudio durante 18 años previos a la realización del estudio, demostraron que marzo presenta una cantidad similar de horas de brillo solar promedio que enero y febrero, meses donde esta especie no se reproduce (Fig. 1). Por lo que la duración del fotoperiodo no parece influenciar el inicio del periodo reproductivo de esta codorniz.

Pese a que la abundancia de sitios para anidar sigue aumentando hasta el fin del periodo lluvioso (obs. pers.), no se observó un aumento en la formación de parejas, ni la presencia de pichones o machos territoriales. Las mayores abundancias se registraron a mediados de la época reproductiva (mayo a julio), cuando aproximadamente el 70\% del área está cubierta por posibles sitios de anidación (obs. pers.). Este patrón es poco común para la mayoría de aves, ya que el periodo donde ocurre la mayor abundancia de parejas, machos territoriales y pichones es al inicio del periodo reproductivo (Howard 1974, Alatalo et al. 1989, Capp 1992, Hoi-Leitner et al. 1995, Kipper et al. 2006). En C. leucopogon podría explicarse este patrón por las características ambientales, ya que antes de mayo los sitios de anidación son escasos y esto puede hacer que las nidadas sean más fácilmente localizables por los depredadores, comparado con la anidación cuando hay una mayor cobertura vegetal (posterior a mayo). Otra posible causa de este patrón, y para que 

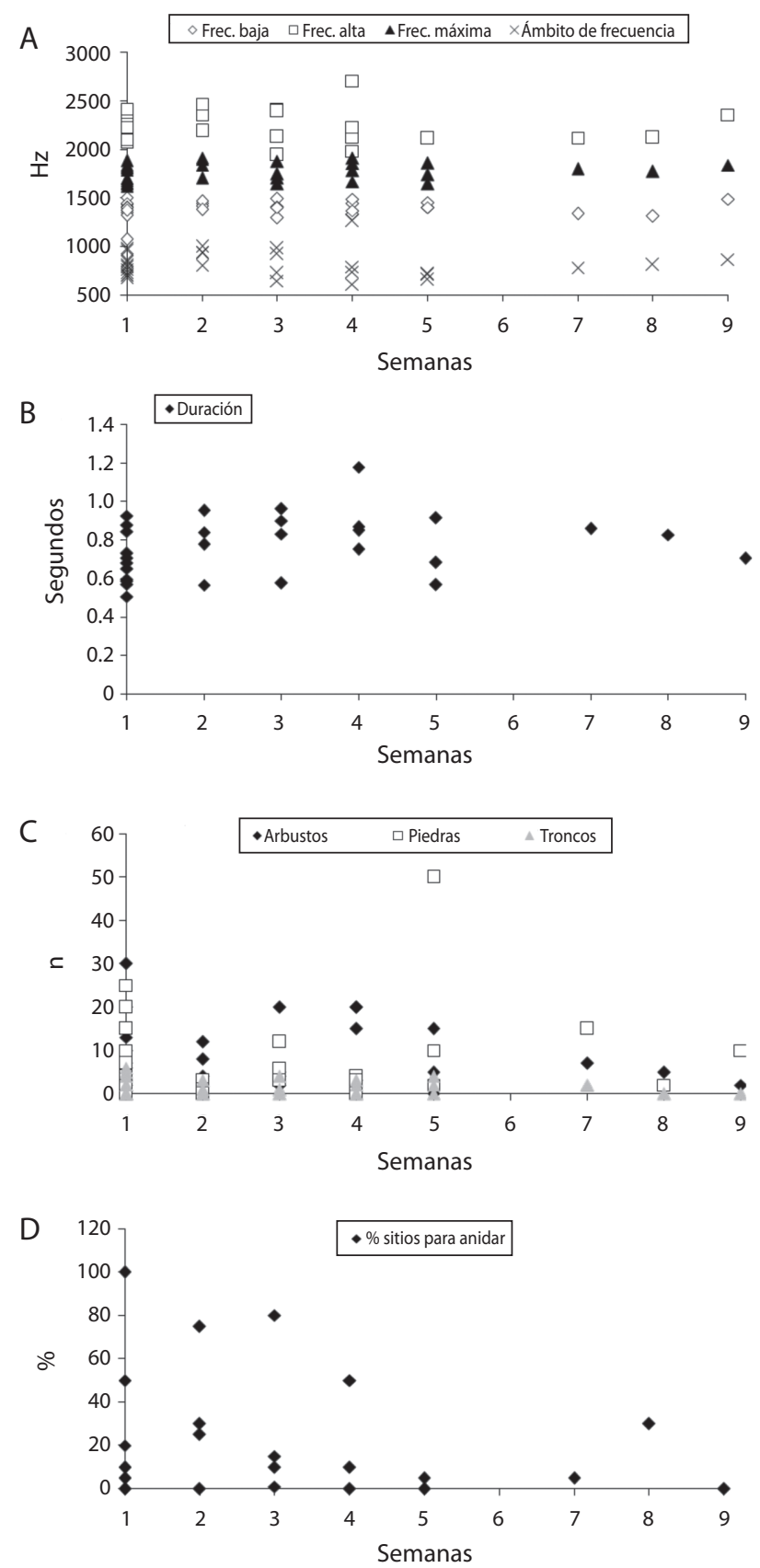

Fig. 5. Correlación de Spearman entre la duración de los machos de Colinus leucopogon en su territorio en semanas durante los periodos reproductivos de 2005-2006, con relación a: (A) los valores de la frecuencia del canto; ( B) la duración del canto; (C) la abundancia de perchas y (D) porcentaje de sitios potenciales de anidación.

Fig. 5. Spearman correlation between the time expended by Colinus leucopogon territorial males, in weeks, during 20052006 reproductive seasons, according to: (A) frequency values of song; (B) song duration; (C) abundance of perches and (D) percentage of possible nesting places. 
la relación observada no se mantuviera hasta el final del periodo reproductivo (agosto a octubre), es que durante estos meses es cuando ocurre la mayor precipitación en el área de estudio (Fig. 1). El aumento de la precipitación puede hacer fracasar los nidos debido a un aumento de parásitos (e.g., hongos o bacterias), asociados con el incremento de la humedad relativa (Stiles 1983, Barrantes \& Sánchez 2003). Además, esta alta precipitación también puede causar un aumento en la mortalidad de los pichones, debido a que durante los primeros días de nacidos, estos poseen una pobre termorregulación (Skutch 1950, Lusk et al. 2001).

Por lo general, en aves que forman parejas durante la época reproductiva, y donde el canto es la característica más importante para la elección de pareja por parte de las hembras como parece ser C. leucopogon, los machos con cantos más atractivos son los primeros en emparejarse (Alatalo et al. 1990, Kipper et al. 2006), por lo que invierten una menor cantidad de tiempo atrayendo hembras y permaneciendo menor tiempo en sus territorios que los machos no seleccionados. Además, dentro de la población de estudio pueden existir machos con características físicas pobres (e.g., poco peso), lo que puede limitar el tiempo que invierten cantando en sus territorios, debido a que el canto es una actividad física de elevado consumo de energía (Eberhardt 1994, Oberweger \& Goller 2001); y además se evita que se realicen otras actividades necesarias para mantener el estado físico de los individuos como la alimentación (Gil \& Gahr 2002). Por lo tanto, la rápida selección de los machos con mejores cantos o la presencia de machos con condiciones físicas pobres pueden estar influenciando la ausencia de relación entre las características medidas del canto y la duración de los machos dentro de sus territorios. Para poder analizar cuál de los dos factores es el más importante explicando el tiempo de permanencia de los machos en su territorio sería necesario pesarlos y marcarlos individualmente. Por otra parte, la falta de relación de las características territoriales con el tiempo de permanencia de los machos en su territorio se puede deber a que todos los machos de C. leucopogon seleccionaron territorios similares a lo largo de la época reproductiva (Sandoval 2008), por lo tanto machos que se emparejaron como los que no lo hicieron, tuvieron territorios con características similares. Otra causa de la falta de relación de la duración de los machos dentro del territorio con las características territoriales puede estar relacionada con que los machos abandonan su territorio después de emparejarse (obs. pers.). Esto posiblemente genera que las características del territorio tengan poca influencia en la formación de las parejas y por lo tanto en la duración dentro de él.

En conclusión, el periodo reproductivo de C. leucopogon está relacionado con un aumento en la precipitación durante el mes de marzo. La mayor cantidad de emparejamientos, número de machos territoriales y parejas con pichones se observaron durante los meses correspondientes a la mitad del periodo reproductivo, cuando existen las mejores condiciones reproductivas en el área. La duración de los machos dentro de su territorio no influye en la adquisición de pareja, ni tampoco está relacionado con las características del territorio o canto de los machos.

\section{AGRADECIMIENTOS}

Agradezco a Idea Wild por financiar el equipo utilizado en esta investigación, y al Instituto Meteorológico Nacional de Costa Rica por facilitarme los datos de la estación de Santa Lucía de Barva. A Gilbert Barrantes, Edgardo Arevalo, William Eberhard, Jack C. Eitniar y Melania Fernández y a tres revisores anónimos por todos los comentarios hechos al borrador de este manuscrito.

\section{RESUMEN}

Los factores que determinan el inicio de la época reproductiva, y la relación entre el tiempo que un macho de Colinus leucopogon defiende su territorio con el éxito reproductivo del mismo son desconocidos. Por lo tanto aquí se reporta el efecto de variables climáticas sobre el inicio de la época reproductiva, y el efecto de la duración de los machos en su territorio con el éxito de adquirir 
pareja. También se analiza la relación entre la duración de los machos en su territorio con las características del canto y el territorio. Precipitaciones superiores a $14.3 \mathrm{~mm}$ en marzo determinaron el inicio de la época reproductiva. Esto favoreció el incremento de alimento y sitios para anidar, lo que podría incrementar el éxito reproductivo de la especie. La duración de los machos dentro de su territorio no se asoció con la adquisición de pareja, debido a que machos que logran emparejarse duraron un tiempo similar en sus territorios, que machos que no se emparejaron. Las características del canto y el territorio tampoco se relacionaron con la duración del macho dentro de su territorio. Por lo tanto esto puede ser otra causa para que no se haya encontrado una relación entre la duración del macho en su territorio con la formación de parejas, ya que las características del canto son las que más influyen en la formación de parejas en esta especie.

Palabras clave: Codorniz de Monte Pechimanchada, época lluviosa, formación de parejas, comportamiento territorial, reproducción, canto.

\section{REFERENCIAS}

Alatalo, R., A. Lundberg \& C. Glynn. 1989. Female pied flycatchers choose territory quality and not male characteristics. Nature 323: 152-153.

Alatalo, R., C. Glynn \& A. Lundberg. 1990. Singing rate and female atracction in thr Pied Flycatcher: an experiment. Anim. Behav. 39: 601-603.

Andersson, M. 1994. Sexual selection. Princeton University, Princeton, Nueva Jersey, EEUU.

Barrantes, G. \& J.E. Sánchez. 2003. Geographical distribution, ecology, and conservation status of Costa Rican dry-forest avifauna, p. 147-159. In G.W. Frankie, A. Mata \& S.B. Vinson (eds.). Biodiversity conservation in Costa Rica. Learning the lessons in a seasonal dry forest. University of California, Los Angeles, California, EEUU.

Barrantes, G., C. Sánchez, B. Hilje \& R. Jaffé. 2008: Male song variation of Green Violetear (Colibri thalassinus) in the Talamanca Mountain Range, Costa Rica. Wilson J. Ornith. 120: 519-524.

Bancroft, G.T., R. Owman \& R. Sawick. 2000. Rainfall, fruiting phenology, and the nesting season of WhiteCrowned Pigeons in the upper Florida Keys. Auk 117: 416-426.

Bonaccorso, E. \& G. Barreto. 2002. Microhabitat use by foraging Venezuelan Wood-Quails (Odontophorus columbianus). J. Field Ornithol. 73: 318-322.
Bosque, C.M., A. Pacheco \& M.A. García-Amado. 2004. The annual cycle of Columbina ground-doves in seasonal savannas of Venezuela. J. Field Ornithol. 75: 1-17.

Capp, M. 1992. Test of the function of the song repertoire in Bobolinks. Condor 94: 468-479.

Catchpole, C.K. \& P.J.B. Slater 1995. Bird song. Cambridge University, Cambridge, Londres, Reino Unido.

Charif, R., C. Clark \& K. Fristrup. 2004. Raven 1.2 User's Manual. Cornell Laboratory of Ornithology, Ithaca, Nueva York, EEUU.

Eberhardt, L.S. 1994. Oxygen consumption during singing by male Carolina Wrens (Thryothorus ludovicianus) Auk 111: 124-130.

Eitniaer, J.C., S.H. Aguilar-Rodríguez, J.T. Baccus \& J.P. Carroll. 2001. Response rate of Bearded WoodPartridges (Dendrortyx barbatus, Aves: Phasianidae) to playback of a recorded call. Vida Silv. Neotrop. 10: 55-57.

Fitzsimmons, L.P., N.K. Barker \& D.J. Mennill. 2008. Individual variation and lek-based vocal distinctiveness in songs of the Screamening Piha (Lipagus vociferans), a suboscine songbird. Auk 125: 908-914.

Gil, D. \& M. Gahr. 2002. The honesty of bird song: multiple constraints for multiple traits. Trends Ecol. Evol. 17: 133-141.

Hale, A. 2006. Group living in the Black-breasted Woodquail and the use of playbacks as a survey technique. Condor 108: 107-119.

Hoi-Leitner, M.,H. Nechtelberger \& H. Hoi. 1995. Song rate as a signal for nest site quality in Blackcaps ( $\mathrm{Syl}$ via atricapilla). Behav. Ecol. Sociobiol. 37: 399-405.

Howard, R. 1974. The influence of sexual selection and interspecific competition on Mockingbird song (Mimus polyglottos). Evolution 28: 428-438.

Johnston, D. 1999. Cool Edit 2000. Syntrillium Software Corporation, Phoenix, Arizona, EEUU.

Kipper, S., R. Mundry, C. Sommer, H. Hultsch \& D. Todt. 2006. Song repertoire size is correlated with body measures and arrival date in common nightingales, Luscinia megarhynchos. Anim. Behav. 71: 211-217.

Leber, K. 1975. Notes on the life history of the Spot-bellied Bobwhite, Colinus leucopogon dickeyi, Conover. Brenesia 5: 7-21. 
Loiselle, B. \& J. Blake. 1990. Diets of understory fruiteating birds in Costa Rica: seasonality and resource abundance. Stud. Avian Biol. 13: 91-103.

Lusk, J., F. Guthery \& S. DeMaso. 2001. Northern Bobwhite (Colinus virginianus) abundant in relation to yearly weather and long-term climate patterns. Ecol. Model. 146: 3-15.

Madge, S. \& P. McGowan. 2002. Pheasants, partridge and grouse. Princeton University, Nueva Jersey, Nueva Jersey, EEUU.

Miller, A.H. 1965. Capacity for photoperiodic response and endogenous factors in the reproductive cycles of an equatorial sparrow. Proc. Natl. Acad. Sci. USA 54: $97-101$.

Oberweger, K. \& F. Goller. 2001. The metabolic cost of birdsong production. J. Exp. Biol. 204: 3379-3388.

Pérez, E. 2000. Seasonal diet composition in the Crested Bobwhite in savannas of central-eastern Venezuela. Stud. Neotrop. Fauna Environm. 35: 91-99.

Poulin, B., G. Lefebvre \& R. McNeil. 1992. Tropical avian phenology in relation to abundance an explotation of food resources. Ecology 73: 2295-2309.

Sandoval, L. 2008. Función del canto y el territorio en machos de la codorniz de monte Colinus leucopogon (Odontophoridae: Aves) durante el periodo reproductivo. Tesis de Maestría, Universidad de Costa Rica, Montes de Oca, Costa Rica.

Seddon, N. \& J.A. Tobias. 2007. Song divergence at the edge of Amazonia: an empirical test of peripatric speciation model. Biol. J. Linnean Soc. 90: 173-188.

Skutch, A. 1950. The nesting seasons of Central American birds in relation to climate and food supply. Ibis 92 : 185-222.

Stiles, F.G. 1983. Birds: introduction, p. 502-530. In Janzen, D.H. (ed.). Costa Rican natural history. Chicago University, Chicago, Illinois, EEUU.

Stiles, F.G. 1992. Effects of a severe drought on the population biology of a tropical hummingbird. Ecology 73: $1375-1390$.

Stiles, F.G. \& A. Skutch. 1989. Guide to the birds of Costa Rica. Cornell University, Ithaca, Nueva York, EEUU.

Vehrencamp, S., J. Bradbury \& R. Gibson. 1989. The energetic cost of display in male sage grouse. Anim. Behav. 38: 885-896.

Voous, K.H. 1950. The breeding seasons of birds in Indonesia. Ibis 92: 279-287.

Wikelski, M., M. Hau \& J.C. Wingfield. 2000. Seasonality of reproduction in a neotropical rain forest bird. Ecology 81: 2458-2472. 